\title{
Avaliação do desempenho do teste rápido de urina no diagnóstico da infeção urinária em idade pediátrica
}

Sofia Cassamo, ${ }^{1}$ Marta Ribeiro, ${ }^{2}$ Leonardo Carneiro, ${ }^{3}$ Susana Castanhinha, ${ }^{4}$ Gabriela Araújo e Sá ${ }^{5}$

\section{RESUMO}

Objetivos: As infeções do trato urinário (ITU) são das infeções bacterianas mais frequentes em pediatria. O exame bacteriológico da urina (EBU) é o teste referência para o diagnóstico de ITU. Sendo um exame moroso, a decisão de iniciar antibioterapia baseia-se na clínica e na urinálise (ASU). A suspeita de ITU na criança e adolescente poderá ser orientada pelo médico de família. O objetivo deste estudo foi avaliar a eficácia da ASU no diagnóstico precoce da ITU em idade pediátrica.

Métodos: Efetuou-se um estudo observacional que incluiu doentes (0-18 anos) que recorreram à urgência pediátrica entre 01/01/2015 e 31/12/2016 e que realizaram colheita de urina para ASU e EBU. Foram analisados os resultados da ASU e comparados com os resultados do EBU. Atribuiu-se diagnóstico definitivo de ITU a todos os doentes com EBU positivo. Determinou-se a sensibilidade, especificidade, valor preditivo positivo (VPP), valor preditivo negativo (VPN) e odds ratio (OR) dos parâmetros nitritos e esterase leucocitária (EL) para o diagnóstico de ITU.

Resultados: Das 3.400 amostras incluídas, 21,2\% tiveram resultado bacteriológico positivo. O microrganismo mais frequente foi Escherichia coli. Os nitritos demonstraram sensibilidade de $28 \%$ e especificidade de $99 \%$ para o diagnóstico. A EL revelou sensibilidade de $90 \%$ e especificidade de $63 \%$. Para o conjunto dos dois, a sensibilidade foi de $27 \%$ e a especificidade de $99 \%$, com VPP 91\% e VPN 83\%. Para EL superior a 500 células/mcl verificou-se especificidade de $93 \%$. No grupo etário 0-12 meses verifica-se um VPP 93\% e VPN $82 \%$ para os dois em conjunto.

Conclusões: Os resultados são concordantes com a literatura: a EL é sensível para o diagnóstico de ITU e os nitritos são específicos. O melhor indicador para exclusão de ITU foi a EL. Estes resultados poderão ser aplicados na consulta aberta de saúde infantil e juvenil ao nível dos cuidados de saúde primários.

Palavras-chave: Infeção urinária; Teste rápido de urina; Urinálise; Urocultura.

\section{INTRODUÇÃO}

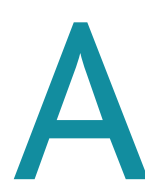
infeção do trato urinário (ITU) caracteriza-se pela invasão e replicação no trato urinário de um agente patogénico. As ITU são uma das infeções bacterianas mais frequentes em idade pediátrica. ${ }^{1}$ Apesar da sua frequência, o diagnóstico de ITU em pediatria pode ser difícil, principalmente na primeira infância, altura em que os sintomas de ITU são frequentemente inespecíficos e não permitem isoladamente localizar a infeção ao trato urinário.

A ITU pode associar-se a complicações graves para o doente a curto e a longo prazo, nomeadamente bacteriemia, urosepsis, lesão renal, insuficiência renal, hi- pertensão arterial. O diagnóstico precoce e a rápida instituição de terapêutica diminuem o risco de complicações, sendo por isso da maior importância..$^{1-2} \mathrm{O}$ exame bacteriológico da urina (EBU) é o teste de referência para o diagnóstico definitivo de ITU $^{2}$ e permite ainda orientar a terapêutica de acordo com o microrganismo

\footnotetext{
1. Médica Interna. USF Rodrigues Miguéis, ACeS Lisboa Norte, Lisboa. 2. Médica Interna. Serviço de Pediatria. Centro Hospitalar Lisboa Norte, Lisboa 3. Médico Interno. Serviço de Patologia Clínica. Centro Hospitalar Lisboa Norte, Lisboa.

4. Médica especialista. Serviço de Urgência Pediátrica, Departamento de Pediatria, Centro Hospitalar Lisboa Norte, Lisboa.

5. Médica consultora. Serviço de Urgência Pediátrica, Departamento de Pediatria, Centro Hospitalar Lisboa Norte, Lisboa.
} 
responsável. Contudo, é necessário um mínimo de 18 horas para detetar crescimento bacteriano e 18 a 24 horas para obter resultados do teste de sensibilidade aos antimicrobianos (TSA). Tendo em conta a importância do início precoce da terapêutica na ITU, a decisão para iniciar antibioterapia empírica baseia-se frequentemente na presença de clínica sugestiva e nos resultados da urinálise (ASU).

A ASU inclui avaliação de parâmetros bioquímicos e microscópicos. A avaliação de parâmetros bioquímicos pode ser realizada através da tira-teste urinária (TTU). A TTU deteta parâmetros como a glicosúria e a proteinúria, bem como a presença de esterase leucocitária (uma enzima produzida pelos leucócitos que, quando detetada, é indicativa da sua presença) e nitritos (que indicam a presença na urina de bactérias redutoras de nitrato) $\cdot^{3-4} \mathrm{O}$ exame do sedimento urinário complementa o TTU e contribui para o diagnóstico de ITU. De acordo com dados da literatura, a presença de esterase leucocitária tem uma sensibilidade de 83\% (67-94\%) para a presença de ITU e uma especificidade de $78 \%$ (64-92\%); a presença de nitritos tem uma sensibilidade de $53 \%$ (15-82\%) e uma especificidade de $98 \%$ (90-100\%). 2,4-5 Na ausência de necessidade de colheita de urina por punção suprapúbica ou algaliação e de critérios clínicos que determinem a referenciação da criança ou adolescente aos cuidados de saúde hospitalares (CSH), ${ }^{6}$ a gestão da ITU poderá ser efetuada pelo médico de família.

A ASU, disponível nos cuidados de saúde primários (CSP), parece constituir uma ferramenta de apoio à decisão de iniciar antibioterapia e/ou necessidade de referenciação aos CSH, contribuindo para a acuidade diagnóstica, tratamento precoce e evicção de terapêutica antibiótica desnecessária.

Com o presente estudo pretende-se avaliar a eficácia da ASU no diagnóstico da ITU em idade pediátrica, com vista à instituição precoce de terapêutica e minimização de complicações ao nível da consulta aberta de saúde infantil e juvenil.

\section{MÉTODOS}

Realizou-se um estudo observacional descritivo, transversal e retrospetivo. Utilizaram-se dados relativos a 2.635 crianças e adolescentes, que recorreram ao Serviço de Urgência Pediátrica (SUP) do Hospital de San- ta Maria (HSM), durante um período de dois anos (entre 1 de janeiro de 2015 e 31 de dezembro de 2016). Foram incluídas no estudo 3.400 amostras, correspondentes às amostras de urina que deram entrada no Laboratório de Urgência (LU) e no Laboratório de Microbiologia (LM), do Serviço de Patologia Clínica do Centro Hospitalar Lisboa Norte (CHLN), no mesmo dia para realização de ASU e EBU, e com resultado válido. Todos os pedidos foram efetuados após observação médica e foram considerados clinicamente justificados, pelo que não se consideraram individualmente critérios clínicos na seleção dos dados. A análise dos dados baseou-se nos dados laboratoriais e demográficos, que foram obtidos a partir do Sistema Informático de Gestão Clinidata ${ }^{\circledR}$ XXI, para a esterase leucocitária e nitritos da ASU na urgência, assim como os resultados dos EBU requisitados pelo SUP.

Excluíram-se amostras contaminadas e com resultado não válido no sistema informático.

O método de colheita da urina correspondeu aos métodos de colheita habitualmente utilizados no SUP do HSM (drenagem vesical por algaliação/caterização, saco coletor, jato médio), escolhido de acordo com a idade e continência de esfíncteres.

As amostras referentes a pedido de ASU enviadas para o LU foram processadas num analisador automatizado de amostras de urina, o Clinitek Atlas ${ }^{\mathrm{TM}}(\mathrm{Sie}-$ mens $^{\mathrm{TM}}$ ), substituído pelo Cobas U $601^{\mathrm{TM}}$ (Roche $^{\mathrm{TM}}$ ) a 30 de julho de 2016. Alternativamente, foi utilizada a TTU Manual Combur Test M Cobas ${ }^{\mathrm{TM}}$ (Roche ${ }^{\mathrm{TM}}$ ), com leitura e interpretação do resultado por técnicos de análises clínicas se quantidade insuficiente de amostra ou indisponibilidade do leitor automatizado. A cultura de urina, com ansa de $10 \mu \mathrm{L}$, foi efetuada em placas de gelose de MacConkey (produzida no laboratório) e gelose de sangue (Biomérieux ${ }^{\mathrm{TM}}$ ). A identificação dos microrganismos nas amostras positivas foi efetuada com recurso ao sistema automatizado de identificação e execução de testes de sensibilidade MicroScan Walkaway ${ }^{\mathrm{TM}}$ (Beckman Coulter ${ }^{\mathrm{TM}}$ ) e, excecionalmente, com recurso a técnicas de identificação manuais.

Os EBU foram classificados como positivos, negativos ou contaminados, de acordo com os critérios de Kass $^{7}$ e outros. ${ }^{8}$ Consideraram-se positivos os exames com presença de um ou dois microrganismos patogénicos numa concentração igual ou superior a 100.000 


\begin{tabular}{|c|c|c|c|}
\hline Grupo etário & Sexo feminino & Sexo masculino & Total \\
\hline [0 - 3 meses] & 87 & 146 & 233 \\
\hline [3 meses - 12 meses] & 224 & 247 & 471 \\
\hline [1 ano - 2 anos] & 194 & 159 & 353 \\
\hline [2 anos - 5 anos] & 517 & 272 & 789 \\
\hline [5 anos - 10 anos] & 446 & 238 & 684 \\
\hline [10 anos - 18 anos] & 685 & 185 & 870 \\
\hline Total & 2.153 & 1.247 & 3.400 \\
\hline
\end{tabular}

unidades formadoras de colónia (UFC) / $\mathrm{ml}$. Consideraram-se negativos aqueles em que não houve crescimento de microrganismos ou em que este foi inferior a 100.000 UFC/ml. Consideraram-se contaminados se crescimento de mais de dois microrganismos ou se existiu crescimento de um ou mais microrganismos identificados com pertencentes à flora da pele ou genitourinária. Alguns casos particulares, por não se enquadrarem nos critérios descritos ou pela existência de dados clínicos adicionais que justificaram atenção particular, foram analisados e classificados individualmente pelo médico patologista, especialista em microbiologia.

Foram determinadas a sensibilidade e especificidade dos parâmetros nitritos e esterase leucocitária que fazem parte daTTU para diagnosticar uma ITU, considerandose a sua existência se o resultado do EBU reportado foi positivo. Determinou-se ainda o valor preditivo positivo (VPP), valor preditivo negativo (VPN) e o odds ratio (OR).

Analisou-se a totalidade dos dados correspondentes às 3.400 amostras em conjunto e também separadamente o grupo de crianças até um ano de idade, considerando que nesta faixa etária os sinais de infeção urinária podem ser mais inespecíficos. Nas duas análises determinou-se a sensibilidade e especificidade, VPP,VPN e OR do parâmetro nitritos separadamente, do parâmetro esterase leucocitária separadamente (considerandose negativos os resultados de esterase leucocitária obtidos com a designação de «negativos» $\mathrm{e}$ «positivos» os resultados com determinação de um valor semiquantitativo para esterase leucocitária, sendo este 15,25, $100 \mathrm{ou}$ 500 células por microlitro), do parâmetro esterase leucocitária separadamente, mas considerando apenas positivas as amostras em que o resultado obtido para a es- terase leucocitária foi de 500 células por microlitro (considerando-se negativos todos os restantes resultados). Analisou-se ainda o desempenho de ambos os resultados em conjunto na previsão de ITU, isto é, nitritos e esterase leucocitária, ambos positivos, e sensibilidade, especificidade, VPP, VPN e OR quando considerado que pelo menos um destes é positivo. Nesta análise conjunta consideraram-se igualmente duas classificações diferentes para esterase positiva: qualquer resultado semiquantitativo $\mathrm{e}$ apenas resultado de 500 células por microlitro.

A análise dos dados foi efetuada com recurso ao programa Excel v. 2013 (Microsoft).

O estudo mereceu parecer favorável da Comissão de Ética do Centro Hospitalar Lisboa Norte e do Centro Académico de Medicina de Lisboa.

\section{RESULTADOS}

A amostra foi constituída por 3.400 amostras de urina, 2.153 provenientes de crianças e adolescentes do sexo feminino e 1.247 do sexo masculino, correspondendo a um total de 2.635 crianças e adolescentes.

Obtiveram-se 721 resultados positivos (21,2\%), 2.536 resultados negativos $(74,6 \%)$ e 143 contaminados $(4,2 \%)$. A mediana de idades foi de 17 anos, com idade mínima de uma semana e máxima de 18 anos (a distribuição por grupo etário encontra-se descrita na Tabela 1).

Os microrganismos mais frequentemente isolados foram Escherichia coli (60,5\%), Proteus mirabilis (15\%), Staphylococcus saprophyticus (7,8\%) e Klebsiella pneumoniae $(6,4 \%)$ (Tabela 2). Isolaram-se duas bactérias em simultâneo em 1,53\% das amostras.

Da análise do parâmetro «nitritos» verificou-se uma sensibilidade de $28 \%$ para o diagnóstico de ITU, uma especificidade de $99 \%$, um VPP de $89 \%$, umVPN de $83 \%$ e um OR de 40,46. A mesma análise efetuada para a esterase leucocitária revelou uma sensibilidade de $90 \%$, uma especificidade de $63 \%$ e um VPP eVPN, respetivamente, de $41 \%$ e $96 \%$, e um OR de 15,41. Considerando os dois parâmetros em conjunto determinou-se uma sensibilidade e uma especificidade no diagnóstico de ITU de $27 \%$ e $99 \%$, respetivamente, com um VPP de $91 \%$, um VPN de $83 \%$ e um OR de 47,3 . Quando considerados resultados de esterase leucocitária semiquantitativos iguais ou superiores a 500 células por microlitro associados à presença de nitritúria, a sensibilidade 
foi de $20 \%$, especificidade de $100 \%$, VPP $94 \%$, VPN $81 \%$ e OR 63,04 (Tabela 3).

A avaliação de desempenho da ASU no grupo etário 0-12 meses revelou uma sensibilidade de $24 \%$ para o parâmetro «nitritos» isoladamente, uma especificidade de $99 \%$, um VPP e VPN de $92 \%$ e $82 \%$, e um OR de 49,89. Para o parâmetro «esterase leucocitária», a sensibilidade foi de $80 \%$ nesta faixa etária, especificidade de $75 \%$, um VPP de $49 \%$, um VPN de $93 \%$ e um OR de 12,51. A avaliação dos dois parâmetros em conjunto demonstrou uma sensibilidade de $24 \%$, especificidade de 99\%, VPP de 93\%, VPN de $82 \%$ e um OR de 62,44 . Quando considerados resultados de esterase leucocitária semiquantitativos iguais ou superiores a 500 células por microlitro associados à presença de nitritúria, a sensibilidade foi de $20 \%$, especificidade de $100 \%$, VPP 98\%, VPN 81\% e OR 193,75 (Tabelas 3 e 4).

\section{DISCUSSÃO}

O parâmetro que demonstrou isoladamente uma maior sensibilidade no diagnóstico de ITU foi a esterase leucocitária, de acordo com a análise dos resultados descritos nas Tabelas 3 e 4. Contudo, a sensibilidade da esterase leucocitária é bastante inferior em crianças com menos de um ano de idade. Em termos de especificidade, o parâmetro «nitritos» demonstrou a mais alta especificidade isoladamente, sendo que em 100 indivíduos com nitritos positivos apenas um não possuía uma ITU. A especificidade dos nitritos é semelhante quando se analisa o grupo com idade inferior a um ano. No entanto, a sensibilidade deste parâmetro quando utilizado isoladamente é reduzida (28\% na amostra total), em particular no subgrupo até um ano de idade, o que se explica por um tempo de permanência vesical da urina baixo nestas idades, insuficiente para a redução dos nitratos a nitritos pela nitrato redutase bacteriana. ${ }^{3}$ Se se considerar em conjunto os indivíduos com nitritos positivos e com um resultado de esterase leucocitária de 500 células por microlitro, o número de indivíduos diagnosticados atinge os $100 \%$ em ambos os grupos, o que é corroborado por um OR de 63,04 e que ainda mais que duplica no subgrupo até um ano de idade (OR 193,75). Por outro lado, verifica-se que o parâmetro «esterase leucocitária» individualmente possui uma especificidade no diagnóstico de apenas $63 \%$ na

\begin{tabular}{|c|c|c|}
\hline Microrganismo & $n$ & $(\%)$ \\
\hline Citrobacter freundii & 4 & 0,55 \\
\hline Citrobacter koseri & 3 & 0,42 \\
\hline Enterobacter cloacae & 4 & 0,55 \\
\hline Enterobacteriaceae & 2 & 0,28 \\
\hline Enterococcus faecalis & 16 & 2,22 \\
\hline Escherichia coli & 436 & 60,47 \\
\hline Klebsiella oxytoca & 5 & 0,69 \\
\hline Klebsiella pneumoniae & 46 & 6,38 \\
\hline Morganella morganii & 2 & 0,28 \\
\hline Proteus mirabilis & 108 & 14,98 \\
\hline Proteus vulgaris & 6 & 0,83 \\
\hline Pseudomonas aeruginosa & 11 & 1,53 \\
\hline Serratia marcescens & 1 & 0,14 \\
\hline Staphylococcus epidermidis & 4 & 0,55 \\
\hline Staphylococcus saprophyticus & 56 & 7,77 \\
\hline Streptococcus Grupo A & 3 & 0,42 \\
\hline Streptococcus Grupo B (S. agalactiae) & 3 & 0,42 \\
\hline Total & 710 & 98,47 \\
\hline Microrganismo & $n$ & $(\%)$ \\
\hline Citrobacter koseri, Staphylococcus saprophyticus & 1 & 0,14 \\
\hline Escherichia coli, Klebsiella oxytoca & 3 & 0,42 \\
\hline Escherichia coli, Staphylococcus saprophyticus & 1 & 0,14 \\
\hline Klebsiella pneumoniae, Escherichia coli & 1 & 0,14 \\
\hline Klebsiella pneumoniae, Proteus mirabilis & 2 & 0,28 \\
\hline Proteus mirabilis, Escherichia coli & 2 & 0,28 \\
\hline Pseudomonas aeruginosa, Enterococcus faecalis & 1 & 0,14 \\
\hline Total & 11 & 1,53 \\
\hline
\end{tabular}

Legenda: $n=$ número de amostras.

amostra total, ou seja, que 37 em cada 100 indivíduos terão um resultado positivo para a esterase leucocitária sem uma ITU. Os resultados obtidos relativamente à sensibilidade e especificidade dos nitritos e esterase leucocitária no diagnóstico de ITU são concordantes com a literatura. ${ }^{2,4-5}$ Neste estudo constatou-se ainda que, quando considerados positivos apenas os valores de es- 


$\begin{aligned} & \text { TABELA 3. Sensibilidade e especificidade da tira reativa de urina na previsão do } \\
& \text { resultado positivo para urocultura, e respetivo valor preditivo positivo e negativo }\end{aligned}$
\begin{tabular}{l|c|c|c|c|c|c|c|} 
[0-18 anos] & N & E & E 500 & N + E & N + E 500 & N ou E & N ou E 500 \\
\hline Sensibilidade & $28 \%$ & $90 \%$ & $58 \%$ & $27 \%$ & $20 \%$ & $91 \%$ & $65 \%$ \\
\hline Especificidade & $99 \%$ & $63 \%$ & $93 \%$ & $99 \%$ & $100 \%$ & $63 \%$ & $93 \%$ \\
\hline VPP & $89 \%$ & $41 \%$ & $70 \%$ & $91 \%$ & $94 \%$ & $41 \%$ & $72 \%$ \\
\hline VPN & $83 \%$ & $96 \%$ & $89 \%$ & $83 \%$ & $81 \%$ & $96 \%$ & $90 \%$ \\
\hline Odds-ratio & 40,46 & 15,41 & 18,41 & 47,30 & 63,04 & 16,32 & 23,67 \\
\hline
\end{tabular}

Legenda: $\mathrm{N}=$ nitritos; $\mathrm{E}=$ esterase leucocitária; $\mathrm{E} 500$ = esterase leucocitária correspondente a 500 leucócitos por microlitro de urina; $\mathrm{N}+\mathrm{E}=$ esterase leucocitária positiva e nitritos positivos; $\mathrm{N}+\mathrm{E} 500=$ nitritos positivos e esterase leucocitária correspondente a 500 leucócitos por microlitro de urina; $\mathrm{N}$ ou $\mathrm{E}=$ nitritos positivos ou esterase leucocitária positiva; $\mathrm{N}$ ou $\mathrm{E} 500$ = nitritos positivos ou esterase leucocitária correspondente a 500 leucócitos por microlitro de urina; $\mathrm{VPP}=$ valor preditivo positivo; $\mathrm{VPN}=$ valor preditivo negativo.

\begin{tabular}{|c|c|c|c|c|c|c|c|}
\hline [0-1 ano] & $\mathbf{N}$ & E & E 500 & $\mathrm{~N}+\mathrm{E}$ & $N+E 500$ & N ou $E$ & N ou E 500 \\
\hline Sensibilidade & $24 \%$ & $80 \%$ & $56 \%$ & $24 \%$ & $20 \%$ & $80 \%$ & $60 \%$ \\
\hline Especificidade & $99 \%$ & $75 \%$ & $97 \%$ & $99 \%$ & $100 \%$ & $75 \%$ & $97 \%$ \\
\hline VPP & $92 \%$ & $49 \%$ & $85 \%$ & $93 \%$ & $98 \%$ & $48 \%$ & $83 \%$ \\
\hline VPN & $82 \%$ & $93 \%$ & $88 \%$ & $82 \%$ & $81 \%$ & $93 \%$ & $89 \%$ \\
\hline Odds-ratio & 49,89 & 12,51 & 41,67 & 62,44 & 193,75 & 12,43 & 42,39 \\
\hline
\end{tabular}

Legenda: $\mathrm{N}=$ nitritos; $\mathrm{E}=$ esterase leucocitária; $\mathrm{E} 500$ = esterase leucocitária correspondente a 500 leucócitos por microlitro de urina; $\mathrm{N}+\mathrm{E}=$ esterase leucocitária positiva e nitritos positivos; $\mathrm{N}+\mathrm{E} 500=$ nitritos positivos e esterase leucocitária correspondente a 500 leucócitos por microlitro de urina; $\mathrm{N}$ ou $\mathrm{E}=$ nitritos positivos ou esterase leucocitária positiva; $\mathrm{N}$ ou $\mathrm{E} 500$ = nitritos positivos ou esterase leucocitária correspondente a 500 leucócitos por microlitro de urina; $\mathrm{VPP}=$ valor preditivo positivo; $\mathrm{VPN}=$ valor preditivo negativo.

terase leucocitária de 500 células por microlitro, a especificidade do parâmetro sobe para os $93 \%$ na amostra total e para $97 \%$ nas crianças até um ano de idade.

Verificou-se ainda que em 100 amostras com nitritos positivos e esterase leucocitária de 500 células por microlitro, 94 terão um resultado positivo no EBU (presumível ITU) (98 para a faixa etária de até um ano de idade). O melhor indicador para exclusão de ITU foi a esterase leucocitária que, quando negativa, exclui um resultado positivo para a EBU em $96 \%$ dos casos (93 no subgrupo de até um ano de idade).
Detetaram-se as seguintes limitações neste estudo:

- Assunção clínica de diagnóstico de ITU exclusivamente com base na presença ou ausência de um resultado positivo do EBU. Podem existir EBU falsamente negativos em situações como utilização de antibioterapia sistémica prévia ou poliúria. Simultaneamente podem existir falsos positivos, nomeadamente dependentes do método da colheita. Numa análise futura poder-se-ia recolher dados em relação a terapêutica antibiótica prévia e eventualmente excluir estas amostras. A metodologia de colheita também deveria ser considerada na análise dos dados. Para perceber a influência da bacteriúria assintomática associada a doença viral febril que leva erradamente a um diagnóstico de ITU, alguns autores sugerem fazer a titulação sérica da proteína $C$ reativa e da procalcitonina em todas as crianças e estudar separadamente aquelas com valores de PCR e procalcitonina baixos. ${ }^{7}$

- Considerar urinas positivas apenas com base nos critérios laboratoriais clássicos, sabendo que se podem ter contaminações mesmo só com um microrganismo e que se podem ter infeções com contagens de colónias inferiores a 100.000 $\mathrm{UFC} / \mathrm{mL}^{2,9}$ A suspeita de ITU baseia-se na clínica, sendo o diagnóstico apoiado nos resultados laboratoriais. A interpretação do resultado da cultura é simples na maioria dos casos, que englobam as culturas sem crescimento e aquelas com contaminação franca. Nos restantes, o critério clássico para classificação de um resultado como positivo (presença de um 
ou dois microrganismos patogénicos numa concentração igual ou superior a $100.000 \mathrm{UFC} / \mathrm{ml}$ ) poderá não considerar todos os resultados positivos, especialmente em idade pediátrica. Os estudos indicam que $30-50 \%$ dos indivíduos com síndroma uretral apresentam culturas com um número de CFU inferior a $100.000 .{ }^{10}$ Mulheres sintomáticas com piúria $\mathrm{e}$ contagens iguais ou superiores a $100 \mathrm{UFC} / \mathrm{ml}$ também foram associadas a possível ITU. ${ }^{11}$ As orientações da American Academy of Pediatric Guidelines sugerem que, entre os dois e os 24 meses de idade, a bacteriúria deve ser valorizada a partir de 50.000 $\mathrm{UFC} / \mathrm{ml}$ de um único microrganismo. ${ }^{4}$ As orientações da European Society for Pediatric Urology referem que qualquer crescimento nas colheitas por punção supra-púbica deve ser considerado como critério de positividade. Nas urinas colhidas por algaliação o limiar a utilizar serão de 1.000 e 100.000 UFC/ml. Na colheita por jato médio, o limiar será superior a 10.000 ou 100.000 , consoante presença ou ausência de sintomas. ${ }^{12}$

- Não foi tido em conta o tempo desde a colheita à cultura de urina nem as condições de refrigeração das amostras. Os estudos indicam que o EBU deve ser efetuado até $4 \mathrm{~h}$ após a colheita. Para além deste tempo, a interpretação de crescimento bacteriano em cultura torna-se progressivamente menos fiável. Quando não for possível a sua execução neste intervalo de tempo, as amostras deverão ser imediatamente refrigeradas. ${ }^{13-14}$

\section{CONCLUSÃO}

Os resultados deste estudo sugerem existir utilidade da ASU no diagnóstico e gestão da ITU na criança. Os dados indicam que a presença de esterase leucocitária e nitritos positivos são o melhor preditor de um resultado positivo na EBU e que um resultado de esterase leucocitária negativo constituirá o melhor indicador para exclusão de ITU. De realçar a relevância do valor semiquantitativo da esterase leucocitária que, quando igual ou superior a 500 células por microlitro e associado a nitritúria, demonstrou uma especificidade de $100 \%$ para o diagnóstico de ITU.

Estes resultados poderão ser aplicados na consulta aberta de saúde infantil e juvenil ao nível dos CSP. De um modo geral, na suspeita de ITU em crianças que não apresentam critérios de gravidade que justifiquem referenciação aos CSH (com quadro séptico, desidratadas, intolerância da via oral, suspeita de pielonefrite abaixo dos seis meses ou antecedentes de patologia nefro-urológica ou quadro séptico), ${ }_{, 5-16}$ a colheita de urina poderá ser efetuada por saco coletor (nas crianças sem controlo de esfíncteres) ou jato médio (na criança e adolescente com controlo de esfíncteres). Perante um resultado de ASU com esterase leucocitária negativa, o diagnóstico de ITU poderá ser excluído com elevada probabilidade e a criança poderá manter-se em vigilância sem necessidade de ser referenciada para realização de EBU. Perante um resultado de esterase leucocitária positiva e nitritos positivos, a probabilidade de se estar perante um diagnóstico de ITU é elevada, tanto mais quanto mais elevado o resultado semiquantitativo da esterase leucocitária na ASU. A criança sem controlo de esfíncteres deverá ser referenciada aos CSH para colheita de urina para EBU por cateterismo vesical ou punção suprapúbica antes de iniciar antibioterapia. $\mathrm{Na}$ criança maior poderá iniciar-se antibioterapia empírica após colheita de urina para realização de EBU em ambulatório. A terapêutica empírica poderá ser posteriormente ajustada de acordo com o antibiograma.

\section{AGRADECIMENTOS}

Ao Professor Ruy Ribeiro (Professor de Bioestatística, da Faculdade de Medicina da Universidade de Lisboa) pelo apoio no tratamento estatístico dos dados.

\section{REFERÊNCIAS BIBLIOGRÁFICAS}

1. Korbel L, Howell M, Spencer JD. The clinical diagnosis and management of urinary tract infections in children and adolescents. Paediatr Int Child Health. 2017;37(4):273-9.

2. National Collaborating Centre forWomen's and Children's Health. Urinary tract infection in children: diagnosis, treatment and long-term management [Internet]. London: RCOG Press; 2007. Available from: http://www.nice.org.uk/guidance/cg54/evidence/full-guideline-pdf-196566877

3. Utsch B, Klaus G. Urinalysis in children and adolescents. Dtsch Arztebl Int. 2014;111(37):617-26.

4. Subcommittee on Urinary Tract Infection, Steering Committee on Quality Improvement and Management. Urinary tract infection: clinical practice guideline for the diagnosis and management of the initial UTI in febrile infants and children 2 to 24 months. Pediatrics. 2011;128(3):595-610.

5. DevilléWL, Yzermans JC, van Duijn NP, Bezemer PD, van der Windt DA, Bouter LM. The urine dipstick test useful to rule out infections: a meta-analysis of the accuracy. BMC Urol. 2004;4:4.

6. Direção-Geral da Saúde. Diagnóstico e tratamento da infeção do trato urinário em idade pediátrica: norma n. ${ }^{\circ}$ 008/2012, de 16/12/2012. Lisboa: DGS; 2012.

7. Kass EH. Asymptomatic infections of the urinary tract. J Urol. 2002;167 (2 Pt 2):1016-9. 
8. Sanford JP, Favour CB, Mao FH, Harrison JH. Evaluation of the positive urine culture: an approach to the differentiation of significant bacteria from contaminants. Am J Med. 1956;20(1):88-93.

9. Tullus K. Difficulties in diagnosing urinary tract infections in small children. Pediatr Nephrol. 2011;26(11):1923-6.

10. Stamm WE, Counts GW, Running KR, Fihn S, Turck M, Holmes KK. Diagnosis of coliform infection in acutely dysuric women. $N$ Eng J Med 1982;307(8):463-8.

11. Nitzan O, Mazen E, Chazan B, Saliba W. Urinary tract infections in patients with type 2 diabetes mellitus: review of prevalence, diagnosis, and management. Diabetes Metab Syndr Obes. 2015;8:129-36.

12. Radmayr C, Bogaert G, Dogan HS, Kocvara R, Nijman JM, Stein R, et al. EAU guidelines on paediatric urology [Internet]. Arnhem: European Association of Urology; 2018. Available from: https://uroweb.org/wp-content/uploads/EAUGuidelines-on-Paediatric-Urology-2018-large-text.pdf

13. Wheldon DB, Slack M. Multiplication of contaminant bacteria in urine and interpretation of delayed culture. J Clin Pathol. 1977;30(7):615-9.

14. Jefferson H, Dalton HP, Escobar MR, Allison MJ. Transportation delay and the microbiological quality of clinical specimens. Am J Clin Pathol. 1975;64(5):689-93.

15. Jones KV.Antimicrobial treatment of urinary tract infection. Arch Dis Child. 1990;65(3):327-30.

16. Shortliffe LM. The management of urinary tract infection in children without urinary tract abnormalities. Urol Clin North Am. 1995;22(1):67-73.

17. National Institute for Health and Care Excellence. Clinical Guideline. Urinary tract infection in under 16s: diagnosis and management (clinical guideline) [Internet]. London: NICE; 2007 [updated 2018 Oct 31]. Available from: https://www.nice.org.uk/guidance/cg54/resources/urinary-tract-infectionin-under-16s-diagnosis-and-management-pdf-975507490501

18. González-Rodríguez JD, Rodríguez-Fernández LM. Infección de vías urinarias en la infancia. Protoc Diagn Ter Pediatr. 2014;1:91-108.

19. Cyriac J, Holden K, Tullus K. How to use... urine dipsticks. Arch Dis Child Educ Pract. 2017;102(3):148-54.

20. Oyaert M, Van Meensel B, Cartuyvels R, Frans J, Laffut W, Vandecandelaere $P$, et al. Laboratory diagnosis of urinary tract infections: towards a BILULU consensus guideline. J Microbiol Methods. 2018;146:92-9.

21. Wilson ML, Gaido L. Laboratory diagnosis of urinary tract infections in adult patients. Clin Infect Dis. 2004;38(8):1150-8.

\section{CONFLITO DE INTERESSES}

Os autores declaram não ter quaisquer conflitos de interesse.

\section{FINANCIAMENTO DO ESTUDO}

Os autores não receberam qualquer financiamento para o desenvolvimento do estudo.

\author{
ENDEREÇO PARA CORRESPONDÊNCIA \\ Sofia Cassamo \\ E-mail: sofiacassamo@gmail.com \\ https://orcid.org/0000-0001-7165-1044
}

Recebido em 15-01-2020

Aceite para publicação em 25-07-2020

\section{ABSTRACT}

\section{PERFORMANCE OF THE DIPSTICK-SCREENING TEST IN URINARY TRACT INFECTION DIAGNOSIS IN CHILDREN}

Objective: Urinary tract infections (UTI) are one of the most common bacterial infections in children. Urine culture (UC) is the reference test for the diagnosis of UTI. Being a lengthy examination, the decision to initiate an antibiotic is based on clinical indicators and urine dipstick screening test (UDST). Management of UTI in the child or adolescent may be carried out by the family doctor. The aim of this study was to evaluate the efficacy of UDST in the early diagnosis of UTI in pediatric age.

Methods: An observational study was carried out. It included patients (0-18 years) who went to the pediatric emergency department between 01/01/2015 and 12/31/2016 and underwent urine collection for ASU and UC. ASU results were analyzed and compared with UC results. A definitive diagnosis of UTI was assigned to all patients with positive UC. Sensitivity, specificity, positive predictive value (PPV), negative predictive value (NPV), and odds ratio (OR) of the nitrite and leukocyte esterase (LE) parameters for the diagnosis of UTI were determined.

Results: Of the 3,400 samples included, $21.2 \%$ had a positive bacteriological result. The most common microorganism was Escherichia coli. Nitrites showed a sensitivity of $28 \%$ and a specificity of $99 \%$ for the diagnosis of UTI. LE revealed a $90 \%$ sensitivity and $63 \%$ specificity. For both, sensitivity was $27 \%$ and specificity $99 \%$, with PPV of $91 \%$ and NPV of $83 \%$. LE was greater than 500 cells/mcl had a $93 \%$ specificity. In the $0-12$-month age group, a PPV of $93 \%$ and NPV of $82 \%$ were found when applying both parameters.

Conclusions: The results are consistent with the literature: LE is sensitive for UTI diagnosis and nitrite is specific. The best indicator for UTI exclusion was LE. These results can be applied in the child and adolescent health visits in primary care.

Keywords: Urinary tract infection; Dipstick; Urinalysis; Urine culture. 